\title{
El sistema espacial de ganadería extensiva del Suroeste de la provincia de Mendoza, Argentina: ¿una región fluida?
}

O sistema espacial de pecuária extensiva do Sudoeste da
província de Mendoza, Argentina: uma região fluida?

Le système spatial d'élevage extensif du Sudouest de la province de Mendoza, Argentine: une région fluide?

\author{
Gloria Leticia Zamorano \\ Universidad Nacional de Cuyo \\ gzamorano01@gmail.com
}

\section{Resumen}

Este trabajo tiene como objetivo estudiar un área marginal del oeste de la Argentina. Se pretende caracterizar el sistema espacial de los pastores trashumantes del suroeste de la provincia de Mendoza y, al mismo tiempo, compararlo con el modelo de regiones fluidas de Armand Frémont (1999), para advertir las semejanzas y diferencias con éste. En el marco de la geografía humanista, se considera el sistema como una región en la cual se reflejan los sentimientos y los valores de los habitantes. Como conclusión, se trata de una región fluida, cuyos pobladores no mantienen lazos profundos con su tierra, sino fuertes vínculos entre sí.

Palabras clave: Mendoza, pastores trashumantes, sistema espacial, región fluida

\section{Resumo}

Este trabalho tem como objetivo estudar uma área marginal do oeste da Argentina. Pretende-se caracterizar o sistema espacial dos pastores transumantes do sudoeste da província de Mendoza e, ao mesmo tempo, fazer a comparação com o modelo das regiões fluidas de Armand Frémont (1999), para perceber as semelhanças e diferenças com este. No marco da geografia humanística, considera-se o sistema como uma região em que se refletem os sentimentos e os 
valores dos habitantes. Como conclusão, trata-se de uma região fluida, onde os povoadores não mantêm laços profundos com a sua terra, mas ligações fortes entre eles.

Palavras-chave: Mendoza, pastores transumantes, sistema espacial, região fluida

\section{Résumé}

L'objectif de ce travail est d'étudier une aire marginale de l'ouest de l'Argentine. On prétend caractériser le système spatial des éleveurs transhumants du Sudouest de la province de Mendoza et, en même temps, le comparer avec le modèle des régions fluides d'Armand Frémont (1999), pour en trouver les ressemblances et les différences avec celui-ci. Dans le cadre de la géographie humaniste, on considère le système comme une région dans laquelle se reflètent les sentiments et les valeurs des habitants. Comme conclusion, il s'agit d'une région fluide, dont les habitants ne maintiennent pas des rapports profonds avec leur terre, mais des liens forts entre eux.

Mots-clés : Mendoza, éleveurs transhumants, système spatial, région fluide

\section{Introducción}

Estudiar en profundidad un amplio territorio del suroeste de la provincia de Mendoza representó para nosotros un gran desafío, no sólo por tratarse de una porción del espacio provincial en el cual vivimos, sino también por la importancia de la comunidad que allí habita: un grupo de pastores trashumantes con costumbres ancestrales.

El territorio que investigamos es marginal en dos sentidos. Por un lado, se trata de una región cuya localización es periférica. Por otro lado, constituye un sistema espacial relativamente aislado del espacio que lo circunda, donde un grupo humano vive según una adaptación precaria a las condiciones biofísicas de su espacio. Éste es el sentido semántico más frecuente del término marginal en la geografía contemporánea.

El interés de los geógrafos por el estudio profundo de estas comunidades se advierte desde la década de 1990, con la revalorización de la identidad de los habitantes de los espacios locales frente a la despersonalización individual en el mundo globalizado. En esa época "es clara la orientación hacia una concepción regional: se trata de delimitar y caracterizar áreas con rasgos marginales en el territorio" (SCHMIDT, 2001, p. 2). A nivel mundial esta preocupación científica se manifiesta, en 1992, en la creación del "Grupo de Estudios sobre Cuestiones de Desarrollo en Regiones Marginales" de la Unión Geográfica Internacional, que se jerarquizó en 1996, cuando pasó a ser la "Comisión sobre la Dinámica de las Regiones Marginales y Críticas". En el ámbito local, dicho interés se materializa en artículos de 
difusión como el de Hortensia Almeida de Gargiulo (1994), quien describe las prácticas colectivas de los habitantes de Malargüe; o bien en trabajos de investigación como el que publicamos en 1999, sobre las fronteras existentes entre los pastores sedentarios y los trashumantes en el sur mendocino, con todo lo que ellas implican en cuanto al arraigo de los pobladores.

Esta tendencia científica se acrecienta en el siglo XXI. En efecto, en la década del 2000 se extiende la temática de las áreas marginales a otros fenómenos que las caracterizan, como la dinámica -poniendo énfasis en el proceso de marginalización-, al mismo tiempo que se enmarca en la evolución de la globalización a nivel mundial. Este interés se acentúa desde 2004, en coincidencia con el cambio de denominación de la comisión de la Unión Geográfica Internacional citada: "Comisión sobre Marginalización, Globalización y Respuestas Regionales y Locales" (SCHMIDT, 2001, p. 2). Se nota una preocupación de los geógrafos por agrupar los estudios locales de comunidades que no viven en una economía de mercado como "regiones marginales", tal como se puede apreciar en el trabajo de José María Señoran (2007), quien expone detalladamente sobre el sistema cultural y la vida de las sociedades pastoriles en el mundo desde la etnoarqueología; o bien en el artículo de Mónica Bendini y otros (2005), quienes analizan las costumbres de los pastores en Neuquén; en el trabajo de Rolando Silla (2005), sobre la comparación de las identidades argentina y chilena en el Alto Neuquén; finalmente, en el libro coordinado por María Eugenia Cepparo (2010), que caracteriza al departamento de Malargüe por sus rasgos de marginalidad.

En este marco de estudios geográficos nos propusimos investigar el suroeste de la provincia de Mendoza, que es marginal tanto por situación periférica -ya que se extiende en un extremo de la superficie provincial, sobre la franja fronteriza argentino-chilena-, como por la adaptación precaria del grupo humano de pastores trashumantes a los condicionamientos naturales del espacio que habitan. Pero en este caso agregamos dos particularidades: por un lado, analizamos el territorio como sistema espacial; por el otro, incorporamos la visión subjetiva de los pobladores, según la óptica de Armand Frémont (1999, p. 189-197).

Nuestra contribución se encuadra, por lo tanto, desde el punto de vista epistemológico, en la geografía humanista, porque permite poner en relación los lugares con la subjetividad de los hombres; analizar las manifestaciones espaciales como resultado de sus prácticas territoriales. De este modo, de acuerdo con Frémont, la región se aprecia como un "espacio vivido", con sus distintos elementos: 
Del hombre a la región y de la región al hombre, las transparencias de la racionalidad son perturbadas por las inercias de las costumbres, los impulsos de la afectividad, los condicionamientos de la cultura, los fantasmas del inconsciente. El "espacio vivido", en toda su amplitud y complejidad, aparece así como el revelador de realidades regionales; éstas tienen sin ninguna duda muchos componentes administrativos, históricos, ecológicos, económicos, pero también, y sobre todo fundamentalmente, psicológicos. (FRÉMONT, 1999, p. 58)

Desde esta óptica, la región constituye un espacio en el cual se reflejan sentimientos y valores de los pobladores, porque es "vista, percibida, sentida, amada o rechazada, modelada por los hombres y proyectando en ellos imágenes que los modelan" (FRÉMONT, 1999, p. 58). El autor citado realiza una clasificación de las regiones en fluidas, arraigadas y funcionales. De acuerdo con esta tipología, el sistema espacial en estudio se correspondería con una región fluida, en la cual sus habitantes no mantienen lazos profundos con su tierra, sino fuertes vínculos entre sí. Como espacio subjetivo, su cohesión se desprende de la integración social, producto de la vida comunitaria que llevan los pobladores, y de la concepción de la familia que resulta de su legado transmitido de generación en generación.

Por otro lado, en cuanto a la metodología, seguimos tanto la marcha hipotético-deductiva como el enfoque sistémico. La aproximación hipotéticodeductiva se desarrolló en tres etapas. En primer lugar, enunciadas las hipótesis que apuntan a describir las pautas del modelo de regiones fluidas de Armad Frémont, se llevó a cabo la recopilación de la información correspondiente, se efectuó la observación del paisaje y se realizaron las entrevistas. Después, en el análisis, ejecutamos el tratamiento de los datos seleccionados: elaboramos los cuadros y tablas de correlación, los gráficos y mapas de apoyo a la investigación. Por último, comparamos los caracteres descubiertos con las pautas propias del modelo y comprobamos o rechazamos las hipótesis de partida, que se desprenden de la siguiente hipótesis general: "El sistema espacial de ganadería trashumante del suroeste de la provincia de Mendoza es una región fluida, según Frémont".

Respecto del enfoque sistémico, nos permitió organizar la investigación para descubrir las características del sistema espacial. Consideramos primeramente que un sistema es:

[...] una entidad autónoma en relación con su entorno, organizada en estructura estable (identificable en la duración), constituida por elementos interdependientes, cuyas interacciones contribuyen 
a mantener la estructura del sistema y a hacerla evolucionar (PUMAIN, 2004, p. 1).

Por lo tanto, un sistema es un conjunto de componentes interrelacionados, abierto hacia el exterior e integrado a otros sistemas menores y mayores, cuya organización estable favorece su evolución.

Ahora bien, en un sistema espacial, de acuerdo con la aproximación estructural-funcionalista, la organización exige dos componentes esenciales: por un lado el espacio, analizado a partir del paisaje como resultado del proceso de creación de las sociedades; por el otro, el tiempo, que sostiene la perennidad de la evolución. Dicho de otro modo, esos dos componentes son: la estructura, que representa el estado o el resultado de la evolución del sistema; la dinámica, que comprende los cambios que dicha evolución supone. Además, ambos aspectos están siempre vinculados entre sí por medio de elementos, algunos previsibles y otros aleatorios.

Por una parte, la estructura abarca cinco componentes esenciales: unidades de apropiación, usos del suelo, lugares centrales, red de comunicaciones y unidades administrativas (PINCHEMEL y PINCHEMEL, 1997, p. 185-186).

Las unidades de apropiación constituyen las porciones del espacio que ocupan las colectividades para desarrollar sus actividades con el objeto de cumplir con su proyecto de vida. Incluyen, por consiguiente, tanto las representaciones sociales que dan lugar a las delimitaciones espaciales, como las condiciones naturales aprovechadas por el grupo humano para satisfacer sus necesidades vitales (figura 1). Las actividades de la población dejan su marca sobre el espacio apropiado en los usos del suelo, como el agrícola, el ganadero, el urbano, que caracterizan a cada sistema espacial cuando predominan en forma notable.

A su vez, las unidades administrativas constituyen las porciones de espacio delimitadas gracias a la gestión gubernamental luego de su apropiación por parte de una colectividad: sus fronteras son más o menos permeables, y pueden ser exteriores o interiores. La gestión se ejerce desde los lugares centrales, que constituyen los espacios más frecuentados por los grupos humanos y están caracterizados por su polaridad. "Son polos de atracción, de difusión, que generan campos de fuerza, de los espacios vectorizados, de las áreas de gravedad." (PINCHEMEL y PINCHEMEL 1997, p. 60). En ese marco, las redes de relaciones -que abarcan todas las redes técnicas y las infraestructuras de comunicaciones invisibles- permiten la circulación de flujos de todo orden entre los lugares centrales del sistema espacial. 


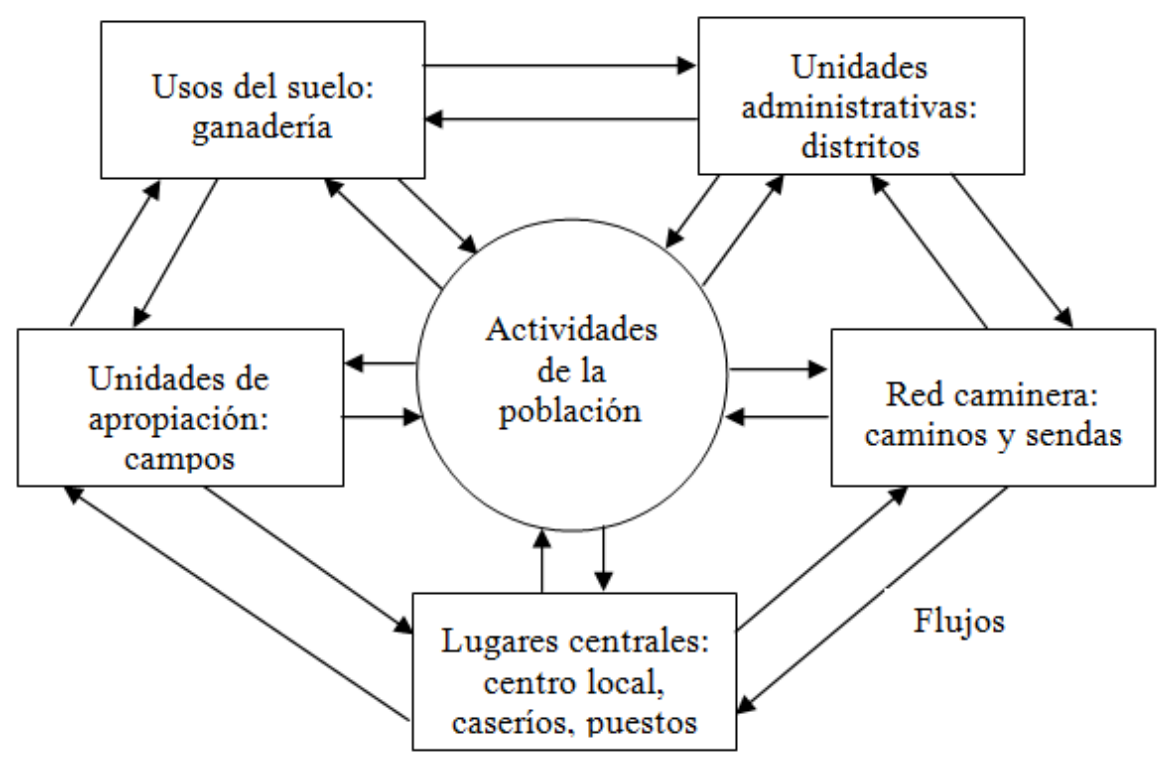

Figura 1. El sistema espacial, con sus componentes.

Fuente: ZAMORANO, 2008.

En suma, dentro de la estructura las unidades administrativas son las superficies controladas por la gestión que la sociedad desarrolla a través de su gobierno -localizado en los lugares centrales- y funcionan gracias al ordenamiento del territorio, plasmado en los distintos usos del suelo interconectados por las redes de relaciones.

Por otra parte, la dinámica es el aspecto evolutivo, temporal, que permite poner en relación los elementos estructurales del sistema espacial. Abarca cuatro componentes esenciales: población, flujos, circuitos de realimentación y plazos de respuesta (ZAMORANO, 2008, p. 19-21).

En primer término, la población incluye a los grupos sociales que reciben las informaciones y las transforman en acciones, permitiendo de este modo la reproducción de los grupos y la creación del espacio. Las acciones o prácticas espaciales involucran tanto la gestión como el ordenamiento del territorio, y tienen como resultado la modificación permanente de los componentes de la estructura y la dinámica del sistema. De este modo lo explica Maryvonne Le Berre: 
El ordenamiento y la gestión del territorio crean entonces a la vez homogeneidad y heterogeneidad territorial: homogeneidad de funcionamiento ligada al objetivo global de mantenimiento en el territorio, y preservada por la cohesión social; heterogeneidad ligada a las acciones diferenciadas que se ejercen sobre lugares específicos (LE BERRE, 1992, p. 633).

El devenir permanente que conlleva la creación de homogeneidad y heterogeneidad territoriales se traduce en los flujos, es decir, los movimientos de población, mercaderías, servicios, energía, capitales, informaciones... desde un punto al otro del sistema, desde y hacia el exterior de éste. Asimismo, el resultado de ese devenir es el proceso de ocupación del suelo, que en el caso de la Argentina implica la apropiación creciente de los distintos espacios hacia todos los puntos cardinales desde el siglo XVI al siglo XXI.

El proceso de ocupación del suelo es viable gracias a la acumulación constante de los circuitos de realimentación, que "tienen por objeto informar a los agentes de decisión acerca de lo que pasa previamente y permitirles ciertamente tomar sus decisiones con conocimiento de causa", según Daniel Durand (1998, p. 13). La retroalimentación implica que los centros de decisión conocen día a día el estado del sistema espacial para actuar sobre él mediante prácticas territoriales (entradas). Si la realimentación es positiva, las acciones tienen como resultado un crecimiento continuo en la producción del espacio, relacionado con el exterior (salidas). Pero si es negativa, el sistema puede desaparecer o bien modificar su objetivo. En los circuitos de retroalimentación son esenciales los plazos de respuesta, es decir, los lapsos de tiempo necesarios para producir un cambio en el sistema. Pueden ser cortos, medianos o largos, según el tipo de problema a resolver. En nuestro estudio no se analizaron los dos últimos componentes mencionados, porque su acción es difícil de descifrar de acuerdo con la información disponible y la metodología de trabajo empleada.

En resumen, los grupos encargados de la gestión toman decisiones para que otros grupos transformen la estructura espacial a través de sus prácticas de ordenamiento del espacio. Así, las acciones se traducen en las configuraciones espaciales que adquieren los componentes estructurales del sistema en distintos plazos de acción: se transforman las unidades administrativas; se dinamizan los lugares centrales, desde y hacia los cuales se dan los flujos; se expanden las redes de relaciones, las superficies apropiadas y los usos del suelo. Por lo tanto, estructura y dinámica están completamente imbricadas en el sistema espacial.

En definitiva, teniendo en cuenta las pautas expuestas acerca de la geografía humanista y la perspectiva sistémica, los objetivos que nos planteamos son: 
$\checkmark \quad$ Caracterizar el sistema espacial de ganadería trashumante del suroeste de la provincia de Mendoza.

Comparar el sistema espacial de los pastores trashumantes con el modelo de regiones fluidas de Frémont, destacando las semejanzas y diferencias.

De acuerdo con estos objetivos tratamos, en la primera parte, los presupuestos teóricos y metodológicos del modelo de regiones fluidas, según Frémont; en la segunda, los caracteres del sistema espacial y la verificación o la refutación de las hipótesis planteadas en la primera parte. Finalmente exponemos las conclusiones.

\section{Primera parte. La aplicación del modelo de regiones fluidas al sistema espacial}

Para Frémont, en una región fluida los hombres no poseen un arraigo fuerte al lugar de su vivienda ni al espacio de sus desplazamientos, sino que mantienen lazos profundos entre sí:

Los lugares existen... Lugares naturales: selva, sabana, colina, vertiente, río... Lugares humanizados: desmontes, recorridos, campamentos, aldeas temporarias o de implantación reciente... Lugares vividos: bosque sagrado, selva temida, campamento familiar... Los hombres también existen, unidos entre sí por estructuras sociales cuya coherencia ha sido subrayada. Pero entre los hombres y los lugares, las relaciones no parecen fijadas de un modo permanente, ya sea que las implantaciones pertenezcan a un pasado reciente, ya sea que las migraciones perduren. En estas condiciones, las regiones no pueden de ninguna manera definirse en un espacio bien delimitado, tan neto en sus contornos como en su duración. La región ciertamente existe, pero con una cierta fluidez (FRÉMONT, 1999, p. 191).

De acuerdo con la interpretación del texto del autor, la región fluida tiene seis caracteres relevantes:

$\checkmark \quad$ Predominio del paisaje natural, alterado por la actividad extensiva del grupo humano, que deja en él huellas poco visibles. Por consiguiente, la densidad de población es baja.

$\checkmark \quad$ La actividad que desarrollan los habitantes se adapta en forma precaria a las condiciones naturales del territorio.

$\checkmark \quad$ Los pobladores realizan sus prácticas territoriales bajo las estructuras de una economía de subsistencia. Por lo tanto, las estructuras 
funcionales de la eco-nomía mercantil pueden superponerse a las de la economía de autoconsumo.

$\checkmark \quad$ El espacio regional no tiene límites bien definidos.

$\checkmark \quad$ Los habitantes son en su mayoría analfabetos; no conocen la escritura.

$\checkmark \quad$ Los medios de transporte son insuficientes en relación con las distancias a recorrer en un medio difícil.

Teniendo en cuenta estos caracteres, planteamos nueve hipótesis relativas al sistema espacial de ganadería trashumante, que después serán confrontadas con el modelo expuesto:

1. El sistema espacial del suroeste de Mendoza presenta un predominio del paisaje natural, con una impronta humana débil.

2. La densidad de población del territorio es baja.

3. La ganadería trashumante se adapta en forma precaria al ecosistema de la Cordillera del Límite, mediante una economía de autoconsumo.

4. La falta de delimitación de los campos en el paisaje indica que los límites de las parcelas de los crianceros no son precisos en su imagen mental.

5. Entre los sistemas de ganadería trashumante del sudoeste y de ganadería sedentaria del sur existe una franja de transición donde cambian los caracteres ecológicos y desde la cual, hacia el oeste, el modo de vida seminómade sustituye al sedentario.

6. Los puesteros o crianceros trashumantes no poseen en su imagen mental una representación exacta del límite occidental de sus desplazamientos.

7. La ciudad de Malargüe y las escuelas albergue son estructuras funcionales de la economía de mercado que se superponen a las estructuras de la economía de subsistencia.

8. Los habitantes son analfabetos, es decir que no conocen la escritura.

9. Los medios de transporte son insuficientes en relación con las distancias a recorrer en un medio difícil. 


\section{Segunda parte. Los caracteres del sistema espacial y la visión de los habitantes}

En este apartado tratamos las características del sistema de ganadería trashumante mencionado, intentando describir sus elementos fundamentales: el proceso de apropiación del suelo con la consecuente formación de las unidades administrativas; los desplazamientos de los pastores y su impronta en el paisaje natural; las unidades de apropiación y su representación por parte de los pobladores; los lugares centrales y su conexión por medio de la red de comunicaciones, que contribuye al desplazamiento de los hombres.

\section{La ocupación progresiva del suelo: conformación de las unidades administrativas}

El sistema espacial en estudio se localiza en el suroeste de la provincia de Mendoza, al oeste de los departamentos de Malargüe y San Rafael: allí se practica la actividad pastoril bajo un sistema bastante precario, con escasa incorporación de tecnología (figura 2). Las unidades administrativas que abarca son los distritos de Ciudad, Río Grande y Río Barrancas en Malargüe; los de Cuadro Benegas y El Nihuil en San Rafael (figura 3). El establecimiento de los puesteros en esos distritos obedece a un proceso de apropiación del suelo que data de 450 años y que se puede dividir en cuatro períodos, destacados por: la conquista de las tierras, la defensa militar del territorio, la instalación de colonias, la vida democrática.

El primer período, de conquista de las tierras, se remonta a la época en que los araucanos tenían sus Malal Hue -de Malal, barda, y Hue, lugar; expresión mapuche de la cual deriva el vocablo Malargüe-, o sea, las empalizadas de piedra, bardas o corrales que les servían a estos indígenas cazadores para guardar sus animales. Hacia 1560, el área fue descubierta y explorada por el español Francisco de Villagra (MUNICIPALIDAD DE MALARGÜE, s./f.). En esa época los mapuches o araucanos, llegados desde Chile, comenzaron su expansión hacia las llanuras y mesetas de la Pampa y la Patagonia. Este fenómeno ocasionó la inestabilidad permanente de las poblaciones fronterizas. Sin embargo, se asistió a una mezcla étnica y cultural de los pueblos nativos argentinos y chilenos con los descendientes de españoles: es la llamada "araucanización". Como efecto de la invasión indígena, el Virreinato del Río de la Plata planeó la creación de una línea defensiva de fuertes y fortines, de localización estratégica. 

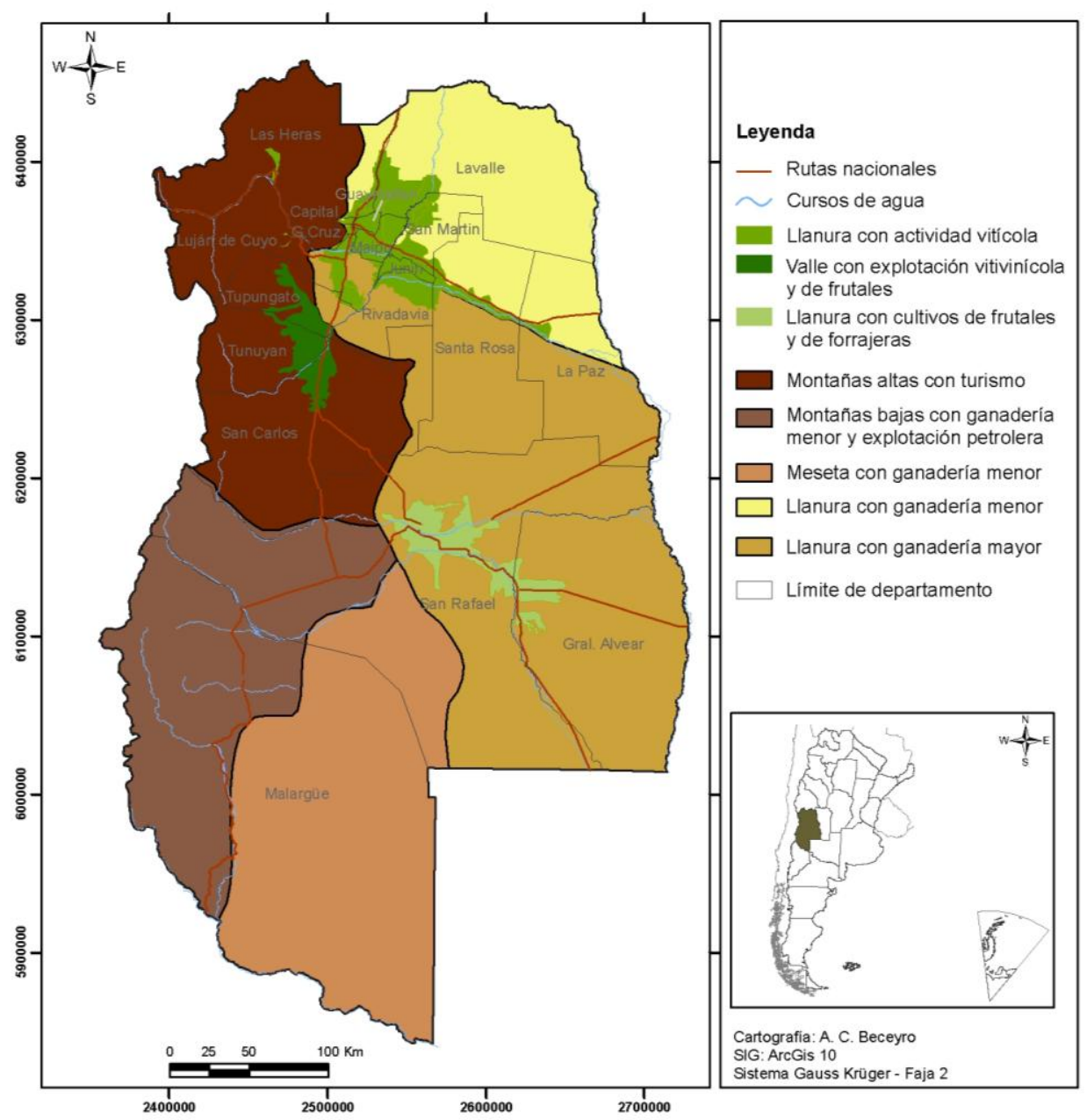

Figura 2. Los sistemas espaciales de la provincia de Mendoza.

Fuente: ZAMORANO, 2013.

El segundo período, de defensa militar del territorio, de 1800 a 1876 , tuvo como objetivo la expansión territorial, desarrollada sobre todo por los efectivos militares. Primeramente los españoles y luego los argentinos construyeron fortificaciones para protegerse contra los ataques de los indígenas. Por ejemplo, el fuerte de San Rafael, denominado Diamante, fue fundado en 1805 junto al río homónimo. Más tarde, en 1846, se creó el fuerte de Malargüe, pero un año después fue fundado el fortín Villa del Milagro, donde se instalaron 120 personas. En 1871, Julio Balloffet construyó el fortín 
Aurora, a tres leguas del fuerte de San Rafael; en 1876 Luis Tejedor creó el fortín San Martín, base de la "conquista del desierto". (SAN RAFAEL TRAVEL, 2007).

El tercer período, de instalación de las colonias, se extiende desde 1877 a 1920. Una vez lograda la expansión hacia la Patagonia, con condiciones de seguridad más estables, continuó la colonización de las tierras del sur de Mendoza. El 30 de abril de 1877, por decisión del gobierno de Mendoza, fue creado el departamento de Malargüe, en un área desértica y con población aborigen, no sujeta al control estatal, que la Nación reclamaba como propia.

El comienzo de la vida democrática marca, para ambos departamentos, el inicio del cuarto período, que se extiende hasta nuestros días. San Rafael se organizó antes: en 1922 tuvo sus primeras elecciones, mientras que en Malargüe éstas comenzaron en 1951. En cuanto a los intercambios internacionales, en 1958 ese departamento tuvo una tentativa de expansión político-económica hacia Chile, cuando se abrió el paso del Pehuenche. Pero poco después, durante los gobiernos militares, la frontera permaneció cerrada, debido a los conflictos con el país vecino.

De este período, en la década de 1960, data la explotación minera en la región. Pero en 1972, debido a la crisis económica argentina, se cerraron algunas de las empresas y aumentó la desocupación. Sin embargo, la actividad fue retomada en 1974: hubo una reactivación petrolera durante veinte años. En el decenio de 1990, las represas hidroeléctricas Nihuil 1, 2, 3 y 4 fueron transferidas a la jurisdicción provincial y posteriormente explotadas por concesión; por otra parte, las empresas petroleras nacionales fueron privatizadas. En consecuencia, se produjo una grave crisis de desindustrialización. Por esta razón, la Municipalidad de Malargüe puso en marcha su Plan Estratégico Malargüe 2000, que abarca a todos los sectores productivos, cuya reactivación comienza a advertirse desde 2002. (CEPPARO et al., 2010, p. 193-213). Por consiguiente, Malargüe tiene un crecimiento inmigratorio sostenido por el aumento de la mano de obra requerida para la explotación petrolera, el turismo, la ganadería -intentando fomentar el aumento de los bovinos-, la agricultura -produciendo papas libres de plagas-. En 2012 las empresas petroleras privadas vuelven a pasar a manos de YPF (Yacimientos Petrolíferos Fiscales), bajo la administración del gobierno nacional, con coparticipación de los gobiernos de las provincias productoras. 


\section{El sistema de ganadería trashumante, con una impronta humana débil}

Los puesteros o crianceros -términos locales que empleamos para designar a los pastores- practican la trashumancia, "movimiento recurrente, pendular y funcional. La periodicidad del movimiento está regulada por el ritmo cíclico de las estaciones y las actividades desarrolladas en las unidades domésticas de producción". (BENDINI, TSAKUMAGKOS y NOGUES, 2005, p. 25). La trashumancia implica entonces el cambio temporario de asentamiento de los pobladores de acuerdo con un ciclo anual de dos momentos, la invernada y la veranada. Según la clasificación de Khazanov (1984), se trata de pastores seminómades, ya que practican una ganadería extensiva realizando una vez al año un desplazamiento en busca de pastos nuevos (SEÑORAN, 2007, p. 3). Se considera una trashumancia vertical, pues consiste en el arreo de los rebaños desde un piso ecológico inferior a uno superior y viceversa: el recorrido se adecua tanto a las condiciones naturales del territorio y como a la receptividad de los campos explotados.

En primer lugar, la explotación extensiva del suelo implica una adaptación precaria o elemental del grupo humano a determinadas condiciones naturales del ambiente, que en este caso son de aridez. Por un lado, la altitud de las formas de relieve es inferior a la del subsistema del noroeste de la provincia de Mendoza, también montañoso. Hacia el occidente, por ejemplo, la cordillera Principal o del Límite no sobrepasa los 5.000 metros de altitud; al noreste, el Macizo Antiguo de San Rafael es aún más bajo, y al sureste, la Depresión Central está ocupada por la laguna de Llancanelo y los ríos Diamante y Atuel (figura 3). Por otro lado, las precipitaciones disminuyen de $900 \mathrm{~mm}^{3}$ anuales en la cima de la montaña a $500 \mathrm{~mm}^{3}$ en el piedemonte. Por sobre 2.500 metros de altura, éstas contribuyen a formar las nieves eternas y a configurar las nacientes de los ríos; a menor altitud aportan humedad al piedemonte y a la depresión central para el crecimiento de las hierbas y los arbustos. La vegetación herbácea aparece sobre todo en los valles y sirve para la alimentación de los rebaños, mientras que la arbustiva, propia de la estepa, está sujeta frecuentemente a la deforestación por parte de los crianceros: su madera es empleada como combustible y para el cierre de los corrales de animales.

En este marco biofísico, a partir de la observación directa, advertimos una fuerte presencia del paisaje natural. En consecuencia, las trazas de la acción humana son visibles, pero muy débiles, sobre todo en los lugares con rebaños, con un hábitat disperso en puestos o caseríos (figura 4). "La naturaleza puede aplastar la presencia de los hombres" (FRÉMONT, 1000, p. 195). 


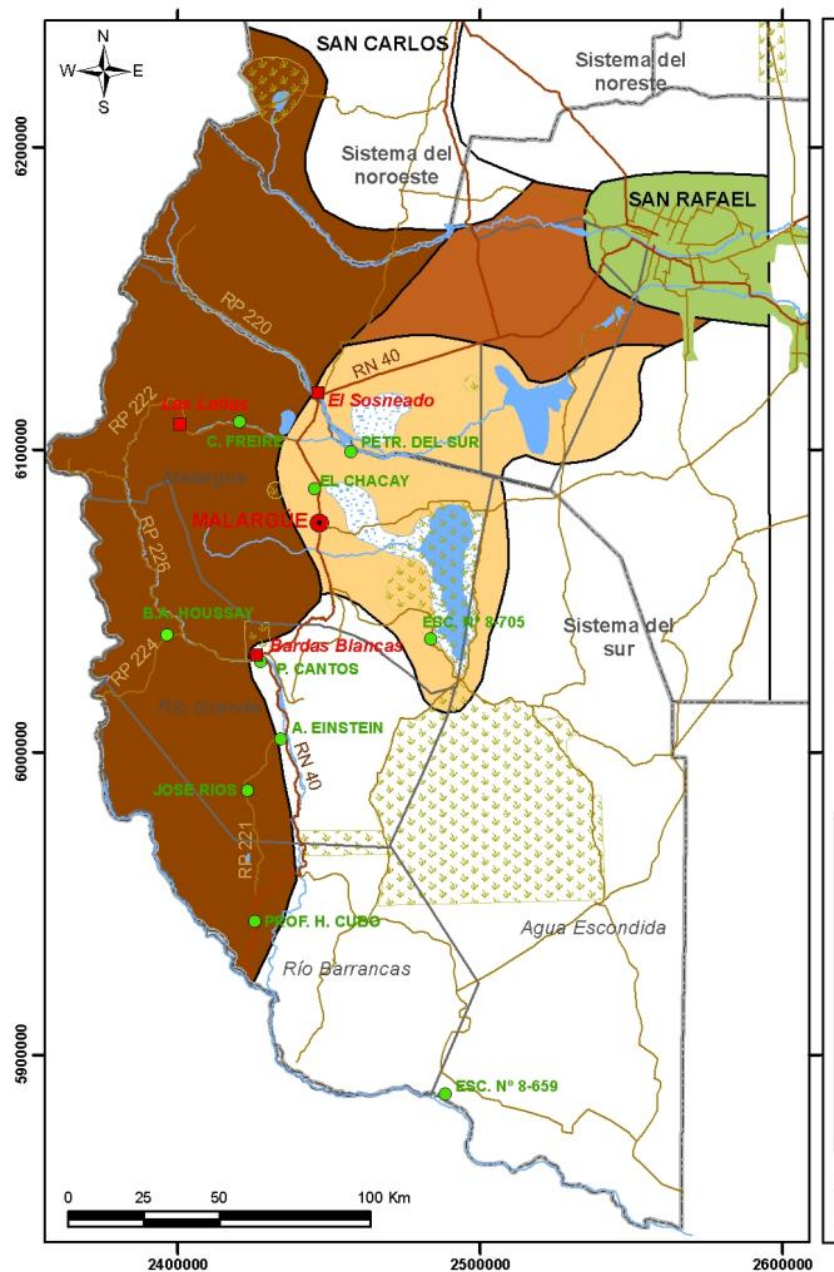

\section{El Sistema Suroeste de ganadería extensiva}

\section{Leyenda}

- Paraje / caserío / localidad

- Ciudad cabecera

- Escuelas albergue/ de frontera

- Rutas nacionales

_ Rutas provinciales

$\sim$ Cursos de agua

Bañados

Embalse / laguna

$x^{x}$ Áreas Protegidas

$\square$ Cordillera Principal

$\square$ Macizo antiguo de San Rafael

$\square$ Depresión Central

$\square$ Sistema de oasis

$\square$ Límite de distrito

$\square$ Límite de departamento

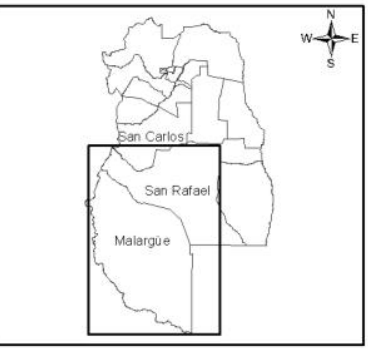

Cartografia: A. C. Beceyro

SIG: ArcGis 10

Sistema Gauss Krüger - Faja 2

Figura 3. Sistema suroeste de ganadería extensiva Fuente: ZAMORANO, 2013.

Esta afirmación de la escasa ocupación humana en un espacio tan inmenso puede ser reforzada por las cifras. En efecto, por un lado, en una superficie de $41.317 \mathrm{~km}^{2}$, el departamento de Malargüe tiene estimativamente, en 2010, 27.727 habitantes; si a esta cifra le restamos los 22.687 pobladores de la ciudad capital homónima, advertimos que 5.040 personas viven en dicho departamento dispersas o agrupadas en pequeños caseríos, lo cual implica una densidad muy baja, de 0,12 habitantes por $\mathrm{km}^{2}$ (tabla 1). Por otro lado, el departamento de San Rafael posee un área de $31.235 \mathrm{~km}^{2}$, ocupada por 188.388 individuos, de los cuales 117.981 se concentran en su ciudad capital; por lo 
tanto, la población dispersa asciende a 70.407 personas y su densidad relativa es también baja, de 2,25 habitantes por $\mathrm{km}^{2}$. Finalmente, en todo el espacio de ambos departamentos, hay unos 75.447 efectivos dispersos en puestos o caseríos, que ocupan una extensión de $72.552 \mathrm{~km}^{2}$, lo cual da una densidad de 1,04 habitantes por $\mathrm{km}^{2}$. (DIRECCIÓN DE ESTADÍSTICAS E INVESTIGACIONES ECONÓMICAS, 2013). Las estadísticas consideradas se aproximan a la realidad, ya que el sistema espacial sólo incluye el oeste del departamento de San Rafael. De todos modos, tanto por medio de la observación directa como a través de las cifras censales verificamos las dos primeras hipótesis:

1. El sistema espacial del suroeste de Mendoza presenta un predominio del paisaje natural, con una impronta humana débil.

2. La densidad de población del territorio es baja.

Tabla 1. Población y densidad del sistema espacial, en 2010

\begin{tabular}{|l|c|c|r|r|r|}
\hline Departamentos & $\begin{array}{c}\text { Población } \\
\text { total }\end{array}$ & $\begin{array}{c}\text { Población en } \\
\text { capital }\end{array}$ & $\begin{array}{c}\text { Población } \\
\text { dispersa }\end{array}$ & Superficie & $\begin{array}{c}\text { Densidad hab./ } \\
\mathrm{km}^{2}\end{array}$ \\
\hline Malargüe & 27.727 & 22.687 & 5.040 & 41.317 & 0,12 \\
\hline San Rafael & 188.388 & 117.981 & 70.407 & 31.235 & 2,25 \\
\hline Total & 216.115 & --- & 75.447 & 72.552 & 1,04 \\
\hline
\end{tabular}

Fuente: DIRECCIÓN DE ESTADÍSTICAS E INVESTIGACIONES ECONÓMICAS, 2010.

En segundo lugar, de acuerdo con la receptividad de los campos, los puesteros se desplazan estacionalmente, en busca de pasturas, desde el piedemonte de las montañas o desde el borde de la meseta de la Payunia hasta la ladera oriental de la cordillera del Límite, a unos 2.200 metros de altitud. La longitud del circuito varía considerablemente, desde unos pocos kilómetros hasta alrededor de 200. "La velocidad de la circulación está condicionada a la alimentación del ganado, a la composición del rodeo, a la cantidad de cabezas, a las características del relieve". (BENDINI, TSAKUMAGKOS y NOGUES, 2005, p. 25-26). El traslado contempla por consiguiente los dos momentos: la invernada, sobre las tierras de mayor degradación natural, y la veranada, sobre los terrenos de mayor receptividad ganadera:

Desde abril a diciembre, aproximadamente, permanecen en la casa que podría ser considerada estable, sobre la llanura, en invernada. Durante este período se produce el apareamiento de los animales y a fines de septiembre comienzan a parir. [...] Aquí las viviendas son confortables, bien terminadas, a menudo de piedra. [...] Esa es "la casa”. (ALMEIDA DE GARGIULO, 1994, p. 57-59) 


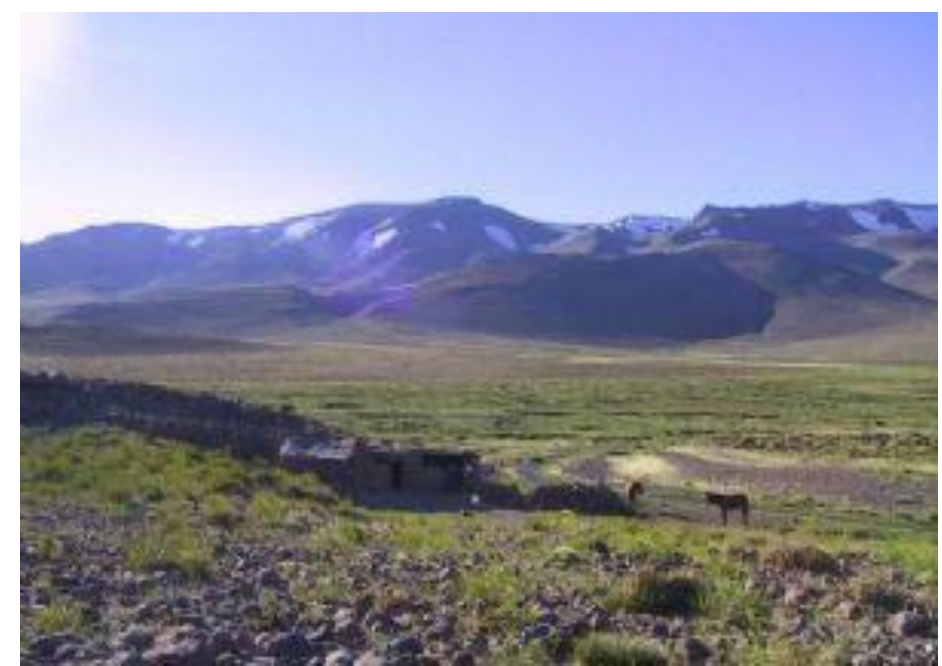

Figura 4. Predominio del paisaje natural en un puesto, 180 $\mathrm{km}$ al sur de Malargüe.

Fuente: http://www.losandes.com.ar/notas/2010/1/3/turismo465103.asp.

A fines de diciembre o principios de enero, cuando las hierbas escasean, el jefe de familia, secundado generalmente por el primogénito y acompañado también por perros adiestrados, parte con las provisiones necesarias hacia las vegas y los valles de la cordillera Principal: allí tienen lugar las "veranadas", que se producen en sitios escarpados, donde pastan los animales. En altura se refugian en grutas naturales o habitan en viviendas precarias hechas de juncos (figura 5).

En suma, por simple observación directa comprobamos que la impronta humana más evidente es la del pastoreo trashumante de cabras y ovejas (tabla 2). Este hecho se verifica también a través de las cifras. En 2011, según la Dirección de Ganadería de la provincia de Mendoza, en Malargüe, 714 crianceros (34\% del total de productores) poseen 594.413 cabras, y 252 puesteros (12\%) crían 106.208 ovejas. Sin embargo, la mayoría de los productores son propietarios de más de un tipo de rebaño, pero en estas estadísticas no está explicitado en qué proporciones. De todos modos, el porcentaje total de cabezas de ganado menor es de $87 \%$, lo cual demuestra que esta actividad predomina en forma notoria en este sistema espacial, y provoca 
un considerable ingreso de divisas en la economía del departamento de Malargüe, según lo expresa el Informe Económico de este municipio:

El movimiento económico interno que genera la ganadería local representa un monto de 281 millones de pesos aproximadamente, de los cuales un porcentaje es utilizado para consumo, otro para crianza y el resto para la venta, esto da un movimiento de 150 millones de pesos aproximadamente en la economía departamental (MUNICIPALIDAD DE MALARGÜE, 2011, p. 1).

Tabla 2. Cantidad de cabezas de ganado, departamento de Malargüe

\begin{tabular}{|l|r|r|r|r|}
\hline $\begin{array}{c}\text { Tipos de } \\
\text { ganado }\end{array}$ & $\begin{array}{c}\text { Cantidad de } \\
\text { cabezas }\end{array}$ & \multicolumn{1}{c|}{$\%$} & \multicolumn{1}{c|}{$\begin{array}{c}\text { Cantidad de } \\
\text { productores }\end{array}$} & \multicolumn{1}{c|}{$\%$} \\
\hline Bovinos & 63.363 & 8 & 420 & 20 \\
\hline Caprinos & 594.413 & 74 & 714 & 34 \\
\hline Ovinos & 106.208 & 13 & 252 & 12 \\
\hline Equinos & 40.003 & 5 & 714 & 34 \\
\hline Total & 803.987 & 100 & 2.100 & 100 \\
\hline
\end{tabular}

Fuente: MUNICIPALIDAD DE MALARGÜE, ÁREA ESTADÍSTICAS, SECRETARÍA DE DESARROLLO ECONÓMICO, 2011, p. 1-2.

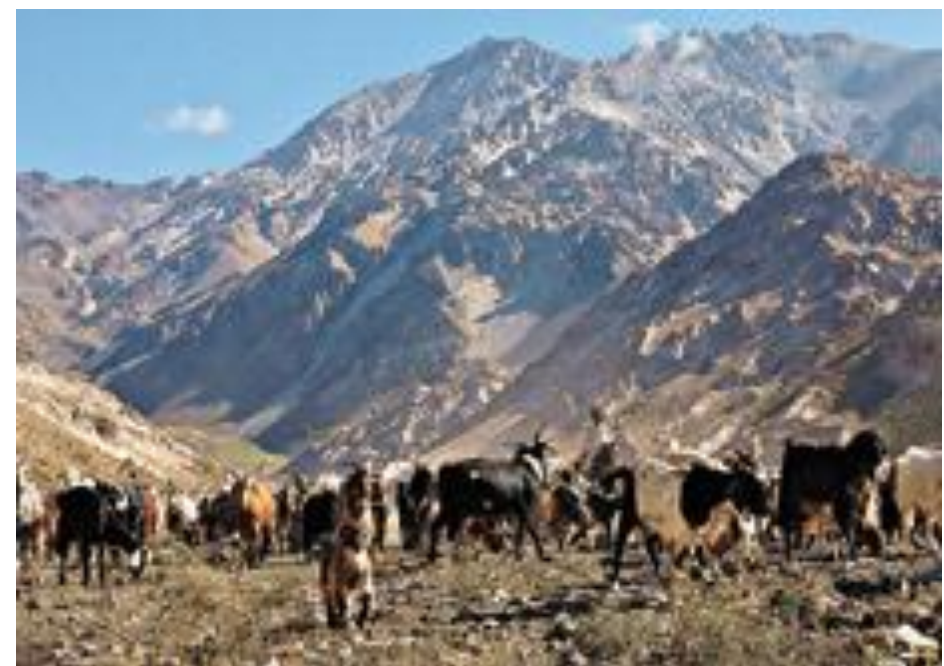

Figura 5. Crianceros en el piedemonte de la Cordillera Principal, de regreso a su casa de invernada.

Fuente: ttp://www.pagina12.com.ar/diario/suplementos/turismo/9-20492011-04-10.html. 
Según las entrevistas, esta utilización del suelo se practica en el marco de una economía de autoabastecimiento. Sin embargo, esta economía no puede ser considerada como totalmente cerrada, porque los crianceros reciben en sus casas a los compradores de sus animales, a los vendedores de mercancías de primera necesidad, o practican el trueque con ellos, o se aprovisionan de productos alimentarios en el caserío más cercano o en Malargüe. En efecto, según Reynaud, se trata de una sociedad aislada, pero relativamente:

\begin{abstract}
$\mathrm{Si}$ un subconjunto territorial no mantiene relaciones con el exterior y vive prácticamente sólo de sus recursos, constituye un subconjunto "aislado". [...] Si estos grupos "aislados" han podido desempeñar históricamente un papel importante en la organización del espacio, en la actualidad constituyen más bien sociedades aisladas relativas... (REYNAUD, 1992, p. 599)
\end{abstract}

De acuerdo con lo expuesto, verificamos la tercera hipótesis, enunciada así: "La ganadería trashumante se adapta en forma precaria al ecosistema de la Cordillera del Límite, mediante una economía de autoconsumo".

Podemos agregar que en este sistema espacial se destacan también otros dos usos del suelo: la explotación petrolera y el turismo, sobre todo gracias a las propiedades de los componentes estructurales, tales como las condiciones climáticas, geomorfológicas, pedológicas, geológicas. Pero estas dos actividades no guardan prácticamente ninguna relación con la vida pastoril extensiva, que es la más antigua.

\title{
Las unidades de apropiación: límites imprecisos ligados a la vida comunitaria
}

Las unidades de apropiación son, en este sistema espacial, los campos de los puesteros, que poseen una configuración particular. Su forma predominante es rectangular, salvo cuando existen ondulaciones topográficas; su extensión no supera normalmente las 1.000 hectáreas: allí se emplazan la casa de invernada y los corrales. La tenencia de estas parcelas es incierta, pues la mayor parte de los crianceros ocupan tierras fiscales y por consiguiente una proporción de ellos no son dueños de sus predios. Pero muchos son propietarios de acuerdo con el derecho de prescripción adquisitiva o posesión veinteañal, según lo manifiesta el código civil argentino en los artículos respectivos. Por consiguiente, en algunos casos se reúnen de ocho a diez propietarios para efectivizar la adquisición, al gobierno o a un propietario 
privado, del predio que ocupa la familia; de este modo economizan los gastos que implica la obtención de sus títulos de propiedad.

El tamaño ideal de sus rodeos, que por lo general son rebaños de cabras y en menor proporción de ovejas, alcanza las 1.000 unidades caprinas, que es el valor de capitalización; pero el valor modal se ubica entre las 250 y 500 unidades. Una pequeña parte de su producción se emplea para el consumo familiar: la leche y la carne sirven para la alimentación del grupo. Pero la mayoría de los "chivos" o de las ovejas se destinan al mercado, principalmente interno. La comercialización de los productos se inserta dentro de la economía informal, con estrategias no registradas y sin continuidad en la producción, que además no está tipificada. Las operaciones comerciales se desarrollan casi siempre en la misma propiedad, ya sea a través del trueque de las cabras u ovejas por productos alimentarios con compradores ambulantes, ya sea con la venta directa de los animales a acopiadores nacionales o a propietarios de hoteles o restaurantes provenientes principalmente de las provincias de Córdoba o San Luis.

El estudio de las imágenes mentales de los puesteros sirve para explicar la problemática de las formas de tenencia y de explotación de los campos, que está vinculada tanto a la fluidez de los contornos de las unidades de apropiación como a la imprecisión de los límites del espacio regional. Consideramos tres tipos de delimitación en relación con las hipótesis enunciadas: los límites de los campos de los crianceros, la frontera entre los dos sistemas ganaderos, y el límite oeste de la región de pastoreo trashumante.

En el primer caso, respecto de los límites de los campos de los crianceros, en el paisaje se observan los alambrados que delimitan las grandes propiedades con extensas superficies desiertas, pertenecientes a propietarios particulares o al Estado. Ahora bien, en cada uno de esos latifundios -de miles de hectáreas incultas- habitan puesteros que comparten con frecuencia la escasa agua disponible. Pero ellos no son propietarios directos de sus tierras, sino ocupantes de hecho o en préstamo. Por esta razón, en la mayoría de los casos no existen señales materiales precisas de los límites de sus predios. Incluso sus rebaños pueden pastar en los campos de los vecinos, sin que esta acción genere algún tipo de conflicto.

Sin embargo, los habitantes poseen -según la información de las entrevistas realizadas- la representación mental de la porción de superficie que les pertenece, de "su" parcela, aunque los límites precisos no estén manifiestos en el paisaje. La imagen mental que ellos tienen se apoya en algunos puntos de referencia, como árboles, cerros, corrales, pozos... Por estas razones, rechazamos la cuarta hipótesis, enunciada como "La falta de delimitación de los campos en el paisaje indica que los límites de las parcelas de los crianceros 
no son precisos en su imagen mental", puesto que los habitantes tienen verdaderamente conciencia de su propio lugar.

No obstante, aquí debemos agregar que los puesteros y sus familias, según la información recogida en las entrevistas - que coincide con las características del modelo de regiones fluidas-, poseen una organización social sólida, basada en sus lazos familiares y comunitarios. Esto se demuestra desde dos ángulos: la organización familiar y la vida comunitaria.

En primer término, en cuanto a la organización familiar, en este grupo de pastores trashumantes, como en otros, se dan dos fenómenos: la patrilinealidad y la patrilocalidad, según Yakar (2000, citado por SEÑORAN, 2007, p. 18). En efecto, se trata de un sistema social en el cual dominan los hombres, y las mujeres están relegadas a un segundo plano. Los varones se encargan del pastoreo y el comercio de productos y subproductos del puesto, mientras que las madres y las hijas recolectan la leña, realizan el ordeñe de los rebaños, fabrican los quesos de cabra o confeccionan artesanías regionales, tales como cueros curtidos o ponchos (SEÑORAN, 2007, p. 28).

Por una parte, según la patrilinealidad, la familia está constituida por el matrimonio y los hijos solteros; el marido es su líder, o sea, quien tiene el control de los recursos. Por lo tanto, como el hombre es quien dirige la sociedad, él es quien aprende las prácticas pastoriles y las transmite a sus descendientes. De allí que casi siempre la herencia pasa al hijo mayor, al primogénito. "La hija sólo recibe la dote cuando se casa. [...]. El hecho de que en algunas familias predominen las hijas supone la pérdida de riqueza de la familia con el pago de las dotes de las hijas..." (SEÑORAN, 2007, p. 18).

Por otra parte, según la patrilocalidad, cuando un hijo se casa, su esposa parte a vivir en la vivienda del marido y su familia, acompañada de la dote que le entregan sus padres. Cuando el número de habitantes de una casa resulta excesivo, el hijo y su esposa se van a habitar una casa anexa o próxima a la de sus padres. Entre los cambios modernos de esta costumbre, producidos sobre todo por la baja rentabilidad de la actividad pastoril y la expansión de la educación femenina, está la migración forzosa de las mujeres jóvenes -en busca de trabajo o por estudio- a los centros urbanos próximos o directamente al Gran Mendoza.

En segundo lugar, con respecto a la vida comunitaria, los puesteros son muy solidarios entre sí. Esto se advierte cuando diferentes miembros de las familias de los crianceros afirman que los límites de las propiedades existen, pero a ellos no les interesan; comparten su predio con los vecinos para hacer pastar a sus rebaños, organizan juntos las fiestas tradicionales, como las carreras de caballos de los domingos en Bardas Blancas (figura 6). Pero algunas fiestas forman parte del circuito comercial y turístico del departamento 
de Malargüe desde hace ya más de dos décadas: la $27^{a}$ Fiesta Nacional del Chivo y $18^{\mathrm{a}}$ Fiesta Provincial del Cordero, organizada por el municipio, se desarrolló en la ciudad cabecera en enero de 2013; la $27^{\mathrm{a}}$ Fiesta Vuelta del Veranador, coordinada por la escuela albergue $\mathrm{N}^{\mathrm{o}}$ 8-511 "Peregrina Cantos" y el mismo municipio, se realizó en Bardas Blancas en abril de 2013; la $1^{\text {a }}$ Fiesta del Puestero Cordillerano fue instituida este año para el mes de marzo, por parte de la Municipalidad de Malargüe -de acuerdo con el nuevo programa "Identidad Puestera"- y la Unión Vecinal Las Loicas, en la localidad homónima (ALSURinforma.com, 2013). En suma, se trata de "un mundo donde el ganado juega un papel especial, tanto a nivel económico como simbólico", pues conduce la vida de los crianceros (SEÑORAN, 2007, p. 29).

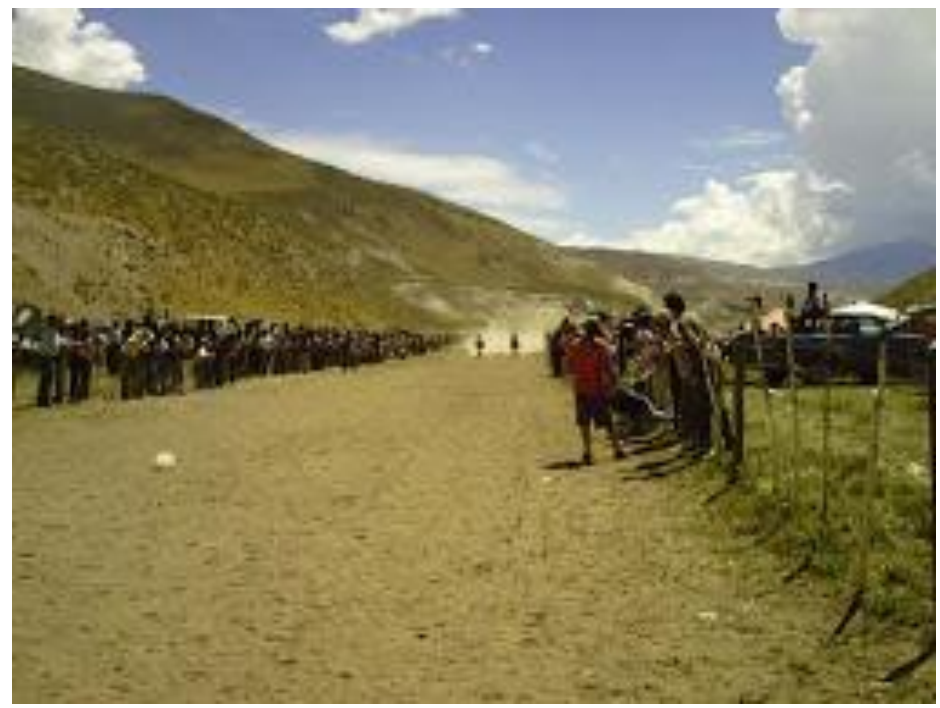

Figura 6. Las carreras "cuadreras" de caballos en Bardas Blancas.

Fuente:http://bardasblancasmisrecuerdos.blogspot.com.ar/2010_03_01_a rchive.html.

En el segundo caso, el límite entre los dos sistemas ganaderos -el de pastoreo sedentario del sur y el de pastoreo trashumante del suroeste-, es la isohipsa de 1.000 metros, es decir, la supuesta línea que, de norte a sur, separa a la meseta de la Payunia del Macizo Antiguo de San Rafael, la depresión Central y el piedemonte de la cordillera Principal (figura 3). Esta delimitación artificial es ciertamente arbitraria por completo, porque no existe una línea 
exacta a partir de la cual pueda producirse un cambio en los componentes de cada sistema, que dé como resultado una transformación abrupta de uno u otro modo de vida.

En contrapartida, los dos sistemas citados están separados por una frontera natural, es decir, una franja de transición o intermedia, que puede ser caracterizada como una zona de contacto de las dos formas de vida. Se trata de una banda a partir de la cual, por el hecho de la adaptación a las condiciones ecológicas, los habitantes de la parte occidental no pueden permanecer todo el año en su vivienda, porque no disponen de pasturas tiernas para los rebaños. Esta frontera es natural, puesto que sólo vemos un cambio de los caracteres biofísicos de los ecosistemas correspondientes.

Pero, por otra parte, existe también una frontera cultural, de origen psicológico, que surge de la representación de los crianceros del sistema de la meseta del sur: "los que practican la trashumancia se encuentran desde el Salitral hacia el oeste", comentan los crianceros entrevistados. Según ellos, entonces, a partir de un borde imaginario situado a unos diez kilómetros hacia el occidente de la laguna de Llancanelo no hay más puesteros sedentarios. Aquí consideramos que la frontera no se percibe como una separación o como un obstáculo, sino como un cambio en la forma de vida.

En resumidas cuentas, se verifica la quinta hipótesis: "Entre los sistemas de montaña del sudoeste y de la meseta del sur existe una franja de transición donde cambian los caracteres ecológicos y desde la cual, hacia el oeste, el modo de vida seminómada sustituye al sedentario". En efecto, los crianceros perciben de una forma imprecisa la delimitación entre las dos regiones, es decir que se trata de una franja de transición y no de una frontera.

Finalmente, a partir de la información resultante de las entrevistas realizadas, afirmamos que los puesteros del sistema montañoso del suroeste no tienen una conciencia precisa del limite occidental de sus desplazamientos. En efecto, la mayor parte de los habitantes explican que, en verano, ascienden hacia la cima de la montaña y llegan hasta "donde empieza el hielo". Este hecho demostraría la falta de precisión en cuanto a la frontera oeste de la región, y nos permite verificar la sexta hipótesis: Los habitantes del triángulo montañoso del suroeste no poseen en su imagen mental una representación exacta del límite occidental de sus desplazamientos.

Si extendemos esta constatación a los otros límites del sistema del suroeste, afirmamos que la representación mental de las fronteras es difusa, como en las regiones fluidas. Esta es una más de las manifestaciones de la fragilidad de los lazos que mantienen estos habitantes con la tierra, y de la fortaleza de sus vínculos sociales. 


\section{Lugares centrales pequeños y aislados, vinculados por rutas y sendas}

Los lugares centrales son, en este sistema, la ciudad de Malargüe y dos pequeños caseríos con muy escasa población (tabla 3 ).

El centro local, Malargüe, es una ciudad que posee, en 2010, una población estimada de 22.687 personas. Su función administrativa es relevante: es la sede del gobierno municipal. También se destaca su función residencial, porque allí se alojan los trabajadores de las empresas petroleras cuyas explotaciones se encuentran a unas decenas de kilómetros, y los turistas que parten diariamente en excursiones a sitios de interés próximos. Tiene, además de las funciones financiera, educativa y médico-asistencial (con un hospital), un equipamiento comercial que abastece a una extensa área rural. Posee un aeropuerto internacional para quienes acceden por vía aérea a la misma ciudad, o al enclave de turismo invernal de Las Leñas -a 75 kilómetros de Malargüecomo destino final. Crece desde el punto de vista científico, ya que en la misma ciudad está instalado, desde 2009, el observatorio Pierre Auger, donde se realizan estudios de rayos cósmicos (OBSERVATORIO PIERRE AUGER, 2009).

Tabla 3. Lugares centrales del sistema de ganadería trashumante

\begin{tabular}{|l|r|c|}
\hline \multicolumn{1}{|c|}{ Nombre de la localidad } & Población estimada 2010 & Jerarquía \\
\hline Malargüe & 22.687 & Centro local \\
\hline El Sosneado & 114 & \multirow{2}{*}{ Caseríos } \\
\hline Bardas Blancas & 79 & \\
\hline Total de población & 28.887 & --- \\
\hline
\end{tabular}

Fuente: elaborado por la autora sobre datos de la D.E.I.E., 2010.

En cambio, los caseríos de Bardas Blancas y El Sosneado proveen de productos de primera necesidad al área rural próxima, pero mientras que El Sosneado presenta un paisaje agreste y una hostería junto a aguas termales no explotadas, Bardas Blancas, sobre la ruta nacional 40, tiene algunas cabañas y hoteles, además de una escuela primaria con un jardín de infantes (figura 7).

También son lugares centrales los establecimientos educativos del departamento de Malargüe, especialmente construidos para los hijos de los 
puesteros. Existen dos escuelas de frontera y ocho escuelas albergue -con una capacidad de alojamiento que oscila entre 40 y 80 alumnos-, que cuentan con los niveles de jardín de infantes y primario (tabla 4, figura 3). Sólo tres establecimientos imparten educación secundaria: en el distrito Malargüe, la escuela No 8-469 Comandante Ramón Freyre, localizada en Los Molles; en el distrito Río Grande, el establecimiento $\mathrm{N}^{\circ}$ 8-497 Bernardo Houssay, ubicado en Las Loicas, y la escuela $N^{\circ}$ 8-513 Alberto Einstein, situada en El Manzano (DIRECCIÓN DE EDUCACIÓN PRIMARIA, GOBIERNO DE MENDOZA, 2007). En esos establecimientos los períodos de clases se ajustan a la rigurosidad del clima; por eso tienen un ritmo de veinte días de trabajo por diez de descanso. Según las entrevistas a los pobladores y de acuerdo con los datos estadísticos, esta región satisface en gran medida las necesidades de formación educativa en los niveles primario y medio.

Por otra parte, la ciudad de Malargüe posee cuatro establecimientos secundarios públicos y cuatro privados, pero no todos los hijos de los crianceros tienen la posibilidad de concurrir diariamente a una escuela de esa ciudad, por el problema de las distancias, que superan por lo general la veintena de kilómetros. Sin embargo, ellos deben ir a Malargüe, a San Rafael o a Mendoza, si desean cursar estudios terciarios o universitarios; también en esas localidades tienen los adultos posibilidades de terminar sus estudios primarios o secundarios (CEPPARO et al., 2010, p. 273-281).

Por todo lo expuesto en relación con el equipamiento urbano y los servicios educativos del sistema, estamos en condiciones de verificar la séptima hipótesis: "La ciudad de Malargüe y las escuelas albergue son estructuras funcionales de la economía de mercado que se superponen a las estructuras de la economía de subsistencia".

Finalmente, para completar los datos sobre educación del grupo humano, consideramos el índice de analfabetismo de la población de 10 años y más, el cual es, en Malargüe, de 8,4\% en 2001 y de 5,6\% en 2010; en San Rafael, de 4,5\% en 2001 y de $2,9 \%$ en 2010 (DIRECCIÓN DE ESTADÍSTICAS E INVESTIGACIONES ECONÓ-MICAS, 2010). Según los datos sobre el equipamiento educativo y el índice de analfabetismo -que está en descenso-, se rechaza la octava hipótesis, que expresa que "los habitantes analfabetos, es decir que no conocen la escritura". Por el contrario, si consideramos que el sistema espacial se localiza en un área de frontera con poblamiento disperso, advertimos que posee un alto índice relativo de alfabetización. 
Tabla 4. Escuelas albergue y de frontera en Malargüe

\begin{tabular}{|c|c|c|c|}
\hline Distrito & Localidad & Nombre de la escuela & Niveles educativos \\
\hline \multirow{4}{*}{ Malargüe } & La Junta & $N^{\circ} 1-374$ Petroleros del Sur & $\begin{array}{l}\text { Jardín de infantes } \\
\text { Primario }\end{array}$ \\
\hline & El Chacay & $N^{\circ} 1-367$ El Chacay & $\begin{array}{l}\text { Jardín de infantes } \\
\text { Primario }\end{array}$ \\
\hline & Los Molles & $\begin{array}{l}\text { No 8-469 Comandante Ramón } \\
\text { Freyre }\end{array}$ & $\begin{array}{l}\text { Jardín de infantes } \\
\text { Primario y secundario }\end{array}$ \\
\hline & Carapacho & $\mathrm{N}^{\mathrm{o}} 8-705 \mathrm{Sin}$ nombre & $\begin{array}{l}\text { Jardín de infantes } \\
\text { Primario }\end{array}$ \\
\hline \multirow{4}{*}{$\begin{array}{l}\text { Río } \\
\text { Grande }\end{array}$} & $\begin{array}{c}\text { El } \\
\text { Alambrado }\end{array}$ & No 8-493 José Ríos & $\begin{array}{l}\text { Jardín de infantes } \\
\text { Primario }\end{array}$ \\
\hline & Las Loicas & No 8-497 Bernardo Houssay & $\begin{array}{l}\text { Jardín de infantes } \\
\text { Primario y secundario }\end{array}$ \\
\hline & $\begin{array}{l}\text { Bardas } \\
\text { Blancas }\end{array}$ & NNo $^{\circ}$-511 Peregrina Cantos & $\begin{array}{l}\text { Jardín de infantes } \\
\text { Primario }\end{array}$ \\
\hline & El Manzano & № 8-513 Alberto Einstein & $\begin{array}{l}\text { Jardín de infantes } \\
\text { Primario y secundario }\end{array}$ \\
\hline \multirow{2}{*}{$\begin{array}{l}\text { Río } \\
\text { Barrancas }\end{array}$} & $\begin{array}{l}\text { Ranquil } \\
\text { Norte }\end{array}$ & No 8-443 Héctor Cubo & $\begin{array}{l}\text { Jardín de infantes } \\
\text { Primario }\end{array}$ \\
\hline & Pata Mora & No 8-659 Río Colorado & $\begin{array}{l}\text { Jardín de infantes } \\
\text { Primario }\end{array}$ \\
\hline
\end{tabular}

El primer número de las escuelas de frontera es el 1; el primero de las escuelas albergue, el 8.

Fuente: DIRECCIÓN DE EDUCACIÓN PRIMARIA, GOBIERNO DE MENDOZA, 2013.

Los lugares centrales se vinculan a través de la red de comunicaciones, que está sobre todo al servicio del turismo y de la explotación petrolera. Constituida por algunas rutas pavimentadas -con una extensión de 270 kilómetros- que bordean los valles longitudinales y algunos caminos de tierra a lo largo de los valles transversales, posee incluso circuitos para visitar los sitios de interés turístico de la región, sean o no importantes. (CEPPARO et al., 2010, p. 225). Cinco ejes primarios del sistema son los más frecuentados; están destinados a las actividades económicas de mercado. Por una parte, la ruta nacional 40 sigue un eje sur-norte junto al río Grande, que separa la cordillera del Límite de la meseta de la Payunia (figuras 3 y 7).

Por otra parte, la ruta provincial 224 -en sentido oeste-este-, muy 
transitada por los habitantes del sur de la provincia, bordea en parte los valles de los ríos Grande y Pehuenche, y comunica con el valle del río Maule en Chile, pero hasta el presente sólo tiene 14 kilómetros de pavimento y aún 32 de tierra; y podrá ser una vía alternativa de la ruta 7 o Panamericana, que sufre en algunas ocasiones grandes embotellamientos de vehículos. En tercer lugar, la ruta provincial pavimentada 222 sirve para el traslado de los turistas desde el aeropuerto internacional de Malargüe a Las Leñas. Por último, la ruta provincial 220 es de tierra y bordea el alto valle del río Atuel, pasa por las termas de El Sosneado y termina en la Planta de Azufre; la ruta provincial 186 es también de tierra y constituye un eje este-oeste que atraviesa la Payunia y vincula a Malargüe con Agua Escondida, junto a la provincia de La Pampa.

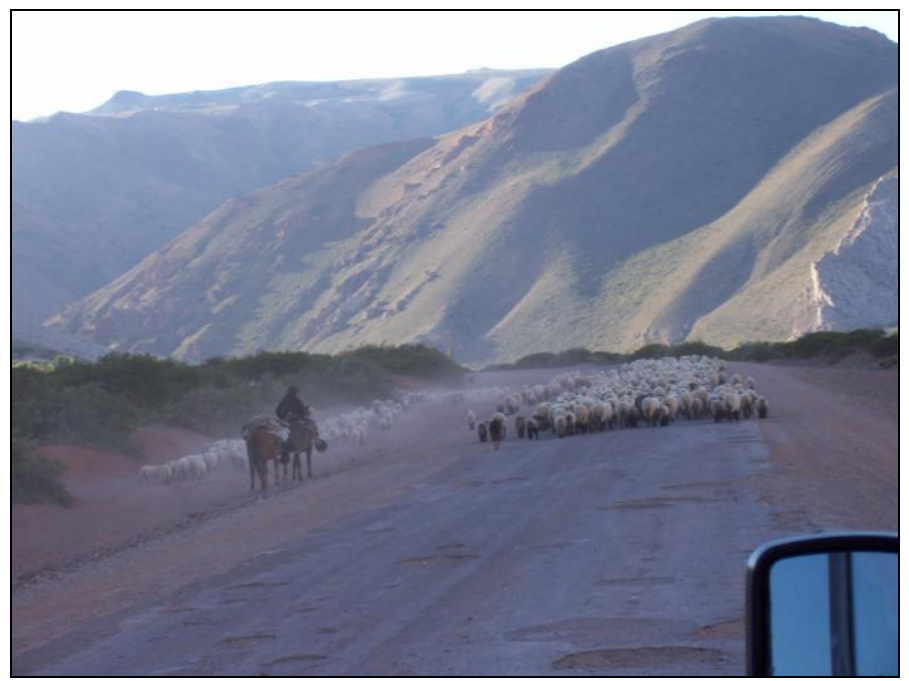

Figura 7. Ruta 40, localidad de Bardas Blancas. Al fondo, la cordillera del Límite.

Fuente: BECEYRO, 2006.

Es necesario agregar que los caminos de tierra que recorren habitualmente las familias de los puesteros son simples sendas, con mal mantenimiento por parte de los organismos responsables de vialidad. Los crianceros se desplazan normalmente por ellas a caballo, para ir de una casa a la otra, de un caserío al otro. Otras veces, cuando andan a pie con sus rebaños, sus perros y sus asnos, toman simplemente huellas.

En resumidas cuentas, la red de comunicaciones que sirve a los pastores es muy pobre. En consecuencia, se verifica la última hipótesis: "Los 
medios de transporte son insuficientes en relación con las distancias a recorrer, en un medio difícil". Sin embargo, cabe aclarar que ni la trama de caminos ni las formas de desplazamiento de los puesteros alteran la calidad de vida de este grupo social, que desarrolla -como dijimos- una adaptación precaria a los condicionamientos naturales.

\section{Conclusiones}

El sistema espacial de ganadería trashumante posee características muy particulares. Se trata de un grupo social descendiente de los primitivos pobladores de Chile, los araucanos, quienes practicaron, desde su instalación e integración con españoles o nativos en tierras mendocinas, la actividad ganadera trashumante presente en sus representaciones sociales. Su modo de vida, con una economía de autoconsumo, se adecua a los caracteres del ecosistema árido con un hábitat disperso en los puestos, con unidades de apropiación en las cuales no interesan prácticamente los límites. Estos fenómenos conllevan las comunicaciones insuficientes y los escasos lugares centrales, a lo cual los habitantes se adaptan de una manera bastante pasiva, sumisa, sin sentir la necesidad de un cambio profundo de su modo de vida.

Con relación a la comparación del sistema espacial con el modelo de Frémont, de las nueve hipótesis que hemos enunciado, siete han sido verificadas y dos refutadas. Las tres primeras pudieron ser comprobadas con la escasa impronta en el paisaje natural que deja este grupo humano, el cual se adapta en forma precaria a las condiciones ambientales gracias a su economía de autoconsumo.

En las otras tres hipótesis, relativas a las unidades de apropiación, advertimos que los pobladores conocen en general la delimitación de sus propiedades, que está presente en su imagen mental, aunque no esté visible en el paisaje. En consecuencia, podemos afirmar que ellos están arraigados a sus tierras de invernada, pero que no tienen apego a los lugares que recorren durante las veranadas: de este modo rechazamos la cuarta hipótesis. En cambio, los habitantes no poseen, en su representación mental, ni el límite entre los dos sistemas ganaderos, ni la frontera occidental del sistema espacial en estudio. Dichos límites tampoco existen como una marca lineal precisa en el paisaje, sino como una franja donde cambian los caracteres ecológicos, y constituyen por lo tanto para los pobladores fronteras culturales, de origen psicológico.

También se comprueba la séptima hipótesis, al observar que tanto el equipamiento y los servicios de la ciudad de Malargüe, como los servicios educativos de las escuelas albergue y de frontera representan estructuras funcionales de la economía de mercado que se superponen a las estructuras de 
la economía de subsistencia del grupo pastoril. Asimismo se verifica la novena hipótesis, puesto que la red de comunicaciones que sirve a los puesteros es muy pobre, con la mayor parte de los caminos en malas condiciones. Por el contrario, la octava hipótesis es rechazada, porque el índice de alfabetización de los crianceros es elevado en relación con el aislamiento del sistema.

En resumidas cuentas, se advierte una analogía relevante entre las características del sistema de ganadería trashumante del suroeste de Mendoza y las pautas planteadas en el modelo de Armand Frémont. La diferencia notable es el alto índice de alfabetización de la población, de lo cual es responsable en gran medida la gestión del gobierno provincial, con el funcionamiento de las diez escuelas del sistema. Además, los habitantes tienen un cierto arraigo a sus tierras de invernada. En consecuencia, estamos en condiciones de verificar la hipótesis general: "El sistema espacial de ganadería trashumante del suroeste de la provincia de Mendoza es una región fluida, según Frémont":

La región ciertamente existe, pero en una cierta fluidez. Fluidez en conexión directa con la que prevalece en las relaciones que unen a los hombres con los lugares. Fluidez, es decir el carácter de lo que, como un líquido, es fácilmente deformable, móvil y cambiante..." (FRÉMONT, 1999, p. 190)

En definitiva, se trata de un sistema espacial cuya cohesión está en el vínculo profundo entre sus pobladores, y no en el apego que ellos tienen hacia las extensiones que recorren en su trashumancia, si bien están arraigados a su puesto de invernada.

\section{Referencias bibliográficas}

ALMEIDA DE GARGIULO Hebe. Proyecto Cuyo. Cuaderno de Cultura Regional, Mendoza, nº 8, 1-59, 1994.

ALSURinforma.com. Se realiza La $1^{\text {a }}$ Fiesta del Puestero Cordillerano. 2013. http://www.alsurinforma.com/16/03/2013/se-realiza-la-1a-fiesta-del-puesterocordillera

BENDINI Mónica, TSAKUMAGKOS Pedro y NOGUES Carlos. Los crianceros trashumantes en Neuquén. In: BENDINI Mónica y ALEMANY Carlos (comp.), Crianceros y chacareros en la Patagonia, Cuaderno GESA 5INTA-NCRCDR. Buenos Aires, La Colmena, 23-40, 2005.

CEPPARO María Eugenia (coord.), PRIETO Estela Beatriz y GABRIELIDIS Graciela (comp.). Rasgos de Marginalidad, diferentes enfoques y aportes para 
abordar su problemática. Malargüe un ejemplo motivador. Mendoza: Editorial de la Facultad de Filosofía y Letras, 2010, 313 p.

DIRECCIÓN DE EDUCACIÓN PRIMARIA, GOBIERNO DE MENDOZA. Las escuelas albergue y de frontera en Malargüe. 2013. In: http://weblog.mendoza.edu.ar/minstitucional/primaria/archives/029583.html.

DIRECCIÓN DE ESTADÍSTICAS E INVESTIGACIONES ECONÓMICAS, GOBIERNO DE MENDOZA. Estadísticas municipales. 2011. In:

http://www.deie.mendoza.gov.ar/publicaciones/detalle_publicaciones.asp?filtro =Publicaciones+Municipales\&id=23.

DIRECCIÓN GENERAL DE ESCUELAS, GOBIERNO DE MENDOZA. Mendoza.edu.ar. Portal Educativo de la Provincia de Mendoza. 2011. In: http://bases.mendoza.edu.ar/intranet/portal_con_esc.asp.

DURAND Daniel. La systémique. 8 ed corregida, París : Presses Universitaires de France, col. "Que sais-je?", 1998, 125 p.

FRÉMONT Armand. La région, espace vécu. 2 ed, París: Flammarion, 1999, 288 p.

GOBIERNO DE MENDOZA. Censo Nacional de Población, Vivienda y Hogares 2010. En: http://www.deie.mendoza.gov.ar/tematicas.

MUNICIPALIDAD DE MALARGÜE, ÁREA DE ESTADÍSTICAS. Informe Ganadería. 2011, 10 p. In:

http://www.malargue.gov.ar/documentos/estadisticas/econom ico/ganaderia/informe-estadistico-dir-ganaderia/ganaderia-estadisticas.pdf.

MUNICIPALIDAD DE MALARGÜE, ÁREA DE ESTADÍSTICAS. Informe Minería. 2011, 10 p. In:

http://www.malargue.gov.ar/documentos/estadististicas/economico/mine ria/informe-estadistico-mineria-malargue.pdf.

MUNICIPALIDAD DE MALARGÜE, CENTRO REGIONAL DE INVESTIGACIÓN Y DESARROLLO CULTURAL. Historia regional. In: http:/www.malargue.gov.ar/hist oria-regional.

OBSERVATORIO PIERRE AUGER SUR. El Observatorio. 2009. In: http://visitantes.auger.org.ar/index.php/el-observatorio/ubicacion-del- 
observatorio.html.

PINCHEMEL Philippe y PINCHEMEL Geneviève. La face de la terre. 5 ed,, París: Armand Colin, 1997, 517 p.

REYNAUD Alain. Centre et périphérie. In: BAILLY Antoine, FERRAS Robert y PUMAIN Denis (coord.). Encyclopédie de la Géographie. París : Economica, 1992, 599-601.

PUMAIN Denise. Système. 2004. In: http://www.hypergeo.eu/, art. 5. SAN RAFAEL TRAVEL. Historia de San Rafael. 2007. In: http://www.sanrafael.travel/Ferrocarril_San_Martin.aspx.

SCHMIDT Margarita Herminia. Marginalidad geográfica: revisión y aplicabilidad de un esquema conceptual mediante la evaluación de estudios de caso desarrollados. In: CONFERENCIA GEOGRÁFICA REGIONAL DE LA UNIÓN GEOGRÁFICA INTERNACIONAL, Comisión 27, Santiago de Chile, 14-18 de noviembre 2011. Conference Proceedings, Santiago de Chile: 2001.110.

SEÑORAN José María. Etnoarqueología de los grupos pastores. Arquoweb, Revista sobre Arqueología en Internet, v. 9, nº 1, 1-30, 2007.

SILLA Rolando. Ambigüedad y superposición de identidades: crianceros argentinos y chilenos en el Alto Neuquén. Anuario de estudios en antropología social. Buenos Aires, Antropofagia, 89-109, 2005.

SUBDIRECCIÓN DE REGALÍAS, GOBIERNO DE MENDOZA. Gestión y Estadísticas, 2011. In: http://www.regalias.mendoza.gov.ar/.

SUBSECRETARÍA DE PROMOCIÓN ECONÓMICA E INVERSIONES, GOBIERNO DE MENDOZA. Petróleo en Mendoza, 2007, 27 p. In: http://energia3.mecon.gov.ar/contenidos/archivos/Reorganizacion/oportunidade s_de_inversion/Mendoza/areas_petroleras.pdf.

ZAMORANO Gloria Leticia. Las fronteras en los geosistemas de ganadería extensiva sedentaria y trashumante en el sur mendocino. Anales de la Sociedad Chilena de Ciencias Geográficas. Santiago de Chile, 443-451, 1999.

ZAMORANO Gloria Leticia. Structures et dynamiques régionales en Argentine: le cas de la province de Mendoza. 2008. Número nacional de tesis: 2007PA010552. Thèse de doctorat en Géographie Nouveau Régime. Lille: 
Université Charles de Gaulle - Lille 3, Atelier National de Reproduction de Thèses, código ISO-8859-1. In:

http://wwwsecheresse.info/article.php3?id_article=7189S. Tesis defendida en el Institut de Géographie, Université de Paris 1, Panthéon Sorbonne, París: el 23/04/20007.

Gloria Leticia Zamorano

Profesora Titular en la Universidad Nacional de Cuyo

Doctora en Geografía de la Universidad de París 1, Panteón Sorbona

Especialista en Cartografía Temática y Analítica de la Universidad de Estrasburgo 1

Remedios Escalada 1606, 5519 Dorrego, Guaymallén, Mendoza, Argentina

Email: gzamorano01@gmail.com

Recebido para publicação em julho de 2013

Aprovado para publicação em agosto de 2013 